\title{
Variability of the adiabatic parameter in monoatomic thermal and non-thermal plasmas ${ }^{\star}$
}

\author{
Miguel A. de Avillez ${ }^{1,2}$, Gervásio J. Anela ${ }^{1}$, and Dieter Breitschwerdt ${ }^{2}$ \\ ${ }^{1}$ Department of Mathematics, University of Évora, R. Romão Ramalho 59, 7000 Évora, Portugal \\ e-mail: mavillez@galaxy.lca.uevora.pt \\ ${ }^{2}$ Zentrum für Astronomie und Astrophysik, Technische Universität Berlin, Hardenbergstrasse 36, 10623 Berlin, Germany
}

Received 4 March 2018 / Accepted 19 April 2018

\begin{abstract}
Context. Numerical models of the evolution of interstellar and integalactic plasmas often assume that the adiabatic parameter $\gamma$ (the ratio of the specific heats) is constant (5/3 in monoatomic plasmas). However, $\gamma$ is determined by the total internal energy of the plasma, which depends on the ionic and excitation state of the plasma. Hence, the adiabatic parameter may not be constant across the range of temperatures available in the interstellar medium.

Aims. We aim to carry out detailed simulations of the thermal evolution of plasmas with Maxwell-Boltzmann and non-thermal $(\kappa$ and $n$ ) electron distributions in order to determine the temperature variability of the total internal energy and of the adiabatic parameter.

Methods. The plasma, composed of $\mathrm{H}, \mathrm{He}, \mathrm{C}, \mathrm{N}, \mathrm{O}, \mathrm{Ne}, \mathrm{Mg}, \mathrm{Si}, \mathrm{S}$, and $\mathrm{Fe}$ atoms and ions, evolves under collisional ionization equilibrium conditions, from an initial temperature of $10^{9} \mathrm{~K}$. The calculations include electron impact ionization, radiative and dielectronic recombinations and line excitation. The ionization structure was calculated solving a system of 112 linear equations using the Gauss elimination method with scaled partial pivoting. Numerical integrations used in the calculation of ionization and excitation rates are carried out using the double-exponential over a semi-finite interval method. In both methods a precision of $10^{-15}$ is adopted.

Results. The total internal energy of the plasma is mainly dominated by the ionization energy for temperatures lower than $8 \times 10^{4} \mathrm{~K}$ with the excitation energy having a contribution of less than one percent. In thermal and non-thermal plasmas composed of $\mathrm{H}$, He, and metals, the adiabatic parameter evolution is determined by the $\mathrm{H}$ and $\mathrm{He}$ ionizations leading to a profile in general having three transitions. However, for $\kappa$ distributed plasmas these three transitions are not observed for $\kappa<15$ and for $\kappa<5$ there are no transitions. In general, $\gamma$ varies from 1.01 to 5/3. Lookup tables of the $\gamma$ parameter are presented as supplementary material.
\end{abstract}

Key words. atomic processes - atomic data - hydrodynamics - methods: numerical - ISM: general - intergalactic medium

\section{Introduction}

Numerical models of the interstellar and galactic media assume that (i) all the gas parcels in the plasma have the same ionic and radiative histories, (ii) the electrons in the plasma have a Maxwell-Boltzmann distribution (hereafter denoted by MB), and (iii) the adiabatic parameter $\gamma$ is a constant.

The first assumption implies that the ionic and radiative histories of the plasma are locked into the cooling function $\Lambda(T)$, which in turn is used as a sink term in the energy equation. $\Lambda(T)$ comprises the loss of energy through radiation by a gas parcel cooling from an initial temperature of $10^{8}-10^{9} \mathrm{~K}$ where it is assumed to be completely ionized and evolves under specific conditions: collisional ionization equilibrium (CIE) or non-equilibrium ionization (NEI; see discussions in, e.g., Shapiro \& Moore 1976; Schmutzler \& Tscharnuter 1993; Sutherland \& Dopita 1993; Gnat \& Sternberg 2007; de Avillez \& Breitschwerdt 2010).

In the interstellar and intergalactic gas simulations at each timestep the temperature of the gas is calculated over the computational domain. For each temperature a value of the cooling

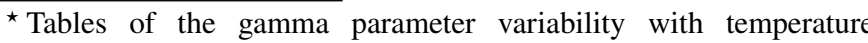
(Fig. 5) + data for $\mathrm{n}=25$ and $\kappa=25$ are only available at the CDS via anonymous ftp to

cdsarc.u-strasbg.fr (130.79.128.5) or via

http://cdsweb.u-strasbg.fr/cgi-bin/qcat?J/A+A/616/A58
}

function is interpolated from lookup tables of $\Lambda(T)\left(\mathrm{erg} \mathrm{cm}^{3} \mathrm{~s}^{-1}\right)$ normalized to $n_{H} n_{e}$ (hydrogen and electron number densities in $\mathrm{cm}^{-3}$ ) previously calculated for an optically thin plasma. Therefore, there is no determination of the ionization structure of the plasma on the fly.

There is mounting evidence that frequently in low density plasmas electrons may be described by non-thermal distributions, for example, $\kappa$ (Vasyliunas 1968), $n$ (Hares et al. 1979; Seely et al. 1987), depleted high energy tail (Druyvesteyn 1930; Behringer \& Fantz 1994), and hybrid MB/power-law tail (Berezhko \& Ellison 1999; Porquet et al. 2001; Dzif̌́áková et al. 2011). These electron distributions occur in any place where a high temperature or density gradient exists, or when energy is deposited into the tail of the distribution at a rate that is sufficiently high to overcome the establishment of thermal equilibrium described by the MB distribution. For applicability in the Astrophysical context, see discussions and references therein in Wannawichian et al. (2003), Karlický et al. (2012), Dzifčáková \& Dudík (2013), Nicholls et al. (2013), Dudík et al. (2014), Humphrey \& Binette (2014), and de Avillez \& Breitschwerdt $(2015,2017)$.

The constancy of the adiabatic parameter used in the simulations is at odds with the ionic evolution of the plasma as its value depends on the plasma internal energy, which includes the contributions from the thermal, ionization and excitation energies 
(see discussions in, e.g., Cox \& Giuli 1968; Schmutzler \& Tscharnuter 1993). Thus, a $\gamma$ parameter consistent with the underlying ionic and radiative histories must be included in these simulations (for different applications of molecular and monoatomic gases see, e.g., Decampli et al. 1978; Wuchterl 1990; D’Angelo \& Bodenheimer 2013; Vandenbroucke et al. 2013; Vaidya et al. 2015). Here we advance previous works by considering the evolution of the adiabatic parameter in plasmas characterized by thermal and non-thermal distributions ( $\kappa$ and $n$ ) and provide lookup tables of the data that can be used in plasma simulations.

The structure of this paper is as follows. Section 2 presents the non-thermal distributions used in the present work, followed by a discussion of the internal energy of a gas parcel in Sect. 3 . Section 4 deals with the framework associated to the thermal model adopted in the present calculations. In Sect. 5 results of the simulations are presented. Section 6 closes the paper with some final remarks. Appendix A describes the tabulated data.

\section{Non-thermal distributions}

The $n$ - and $\kappa$-distributions in the energy space have the analytical forms (Hares et al. 1979; Seely et al. 1987)

$f_{n}(E) \mathrm{d} E=\frac{2}{\sqrt{\pi}\left(k_{\mathrm{B}} T\right)^{3 / 2}} B_{n} E^{1 / 2}\left(\frac{E}{k_{\mathrm{B}} T}\right)^{(n-1) / 2} \mathrm{e}^{-E / k_{\mathrm{B}} T} \mathrm{~d} E$,

with

$B_{n}=\frac{\sqrt{\pi}}{2 \Gamma(n / 2+1)} \quad$ and $\quad n \in[1,+\infty]$.

and (Livadiotis \& McComas 2009; Pierrard \& Lazar 2010)

$f_{\kappa}(E) \mathrm{d} E=\frac{2 E^{1 / 2}}{\pi^{1 / 2}\left(k_{\mathrm{B}} T\right)^{3 / 2}} A_{\kappa}\left[1+\frac{E}{(\kappa-3 / 2) k_{\mathrm{B}} T}\right]^{-\kappa-1} \mathrm{~d} E$,

with

$A_{\kappa}=\frac{\Gamma(\kappa+1)}{\Gamma(\kappa-1 / 2)(\kappa-3 / 2)^{3 / 2}} \quad$ and $\quad \kappa \in[3 / 2,+\infty]$,

respectively. In these expressions $E$ is the electron energy $(\mathrm{eV})$,

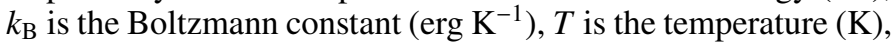
and $\Gamma(x)$ is the Gamma function of variable $x$.

The $n$-distribution (which becomes the MB distribution when $n=1$ ) is characterized by having a mean energy that depends on the $n$ parameter being given by $\langle E\rangle=(3 / 2) k_{\mathrm{B}} \tau$ where $\tau=T(n+2) / 3$ is a pseudo-temperature. This means that at $\tau$ the mean energies of the $n$ - and MB distributions are the same. Thus, $\tau$ has the same physical meaning as $T$ in the MB distribution. The $n$-distributions with the same $\tau$ have their peaks higher and narrower than that of the MB distribution, that is, they have less electrons with both high and low energies, but have an increased number of electrons with intermediate energies (top panel of Fig. 1).

The $\kappa$-distribution is characterized by a high-energy powerlaw tail and having a mean energy that does not depend on $k$ and is given by $\langle E\rangle=3 / 2 k_{\mathrm{B}} T$. Hence, $T$ can be defined as the thermodynamic temperature for these distributions. When $\kappa \rightarrow \infty$, the MB distribution is recovered. As $\kappa$ decreases, deviations from the MB distribution increase, reaching a maximum when $\kappa$ approaches 3/2 (bottom panel of Fig. 1).
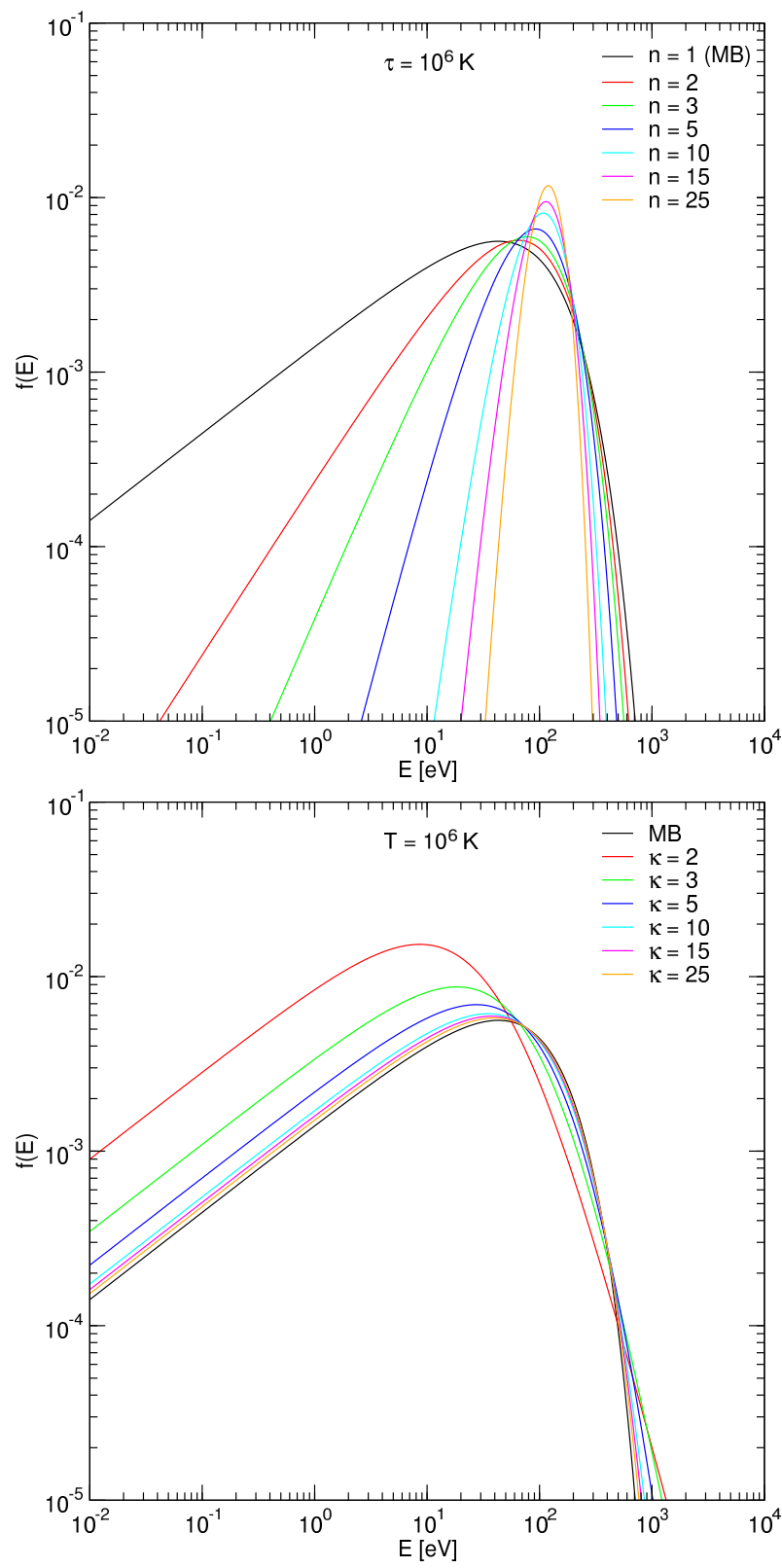

Fig. 1. Maxwell-Boltzmann (both panels), $n$ (top panel) and $\kappa$ (bottom panel) distributions at $\tau, T=10^{6} \mathrm{~K}$. The $n>1$ distributions have a steeper high-energy tail than the MB distribution. The $\kappa$ distribution approaches MB when $\kappa \rightarrow \infty$.

\section{Internal energy and the adiabatic parameter $\gamma$}

The internal energy of the gas parcel includes the contributions due to the thermal translational energy plus the energy stored in (or delivered) from the high ionization stages and from the excitation levels (see, e.g., Cox \& Giuli 1968; Macfarlane 1989; Schmutzler \& Tscharnuter 1993)

$\rho u=\frac{3}{2}\left(n_{\mathrm{tot}}+n_{e}\right) k_{\mathrm{B}} T+E_{s i}+E_{s e} \operatorname{erg} \mathrm{cm}^{-3}$,

with $\rho, u, k_{\mathrm{B}}$, and $T$ denoting the gas density $\left(\mathrm{g} \mathrm{cm}^{-3}\right)$, the specific energy of the gas (erg $\mathrm{g}^{-1}$ ), respectively; $n_{\mathrm{tot}}$ and $n_{e}$ are the total number density of the element with atomic number $Z$ (hereafter referred to as element $Z$ ) and the electron density, 
respectively, and are given by

$n_{\mathrm{tot}}=\sum_{Z} n_{z}=\sum_{Z} \sum_{z=0}^{Z} n_{Z, z} \quad$ and $n_{e}=\sum_{Z} \sum_{z=1}^{Z} z n_{Z, z}$,

where $z$ is the ion state, and $n_{z}$ and $n_{z, z}$ are the number densities of the element $Z$ and of its ionic state $z$. The energies stored in ionization and in excitation are, respectively,

$E_{s i}=\sum_{Z} \sum_{z=1}^{Z} n_{Z, z}\left(\sum_{l=0}^{z-1} \Phi_{Z, l}\right) \operatorname{erg~\mathrm {cm}^{-3}}$

and

$E_{s e}=\sum_{Z} \sum_{z=1}^{Z}\left[\sum_{j=n_{o}+1} n_{z, z, j}\left(\Delta E_{j, n_{o}}\right)_{Z, z}\right] \operatorname{erg~\mathrm {cm}^{-3}.}$

In these equations $\Phi_{Z, l}$ is the ionization potential (erg) of the ionization state $l$ of the element $Z$ and $\Delta E_{j, n}$ is the excitation energy (erg) with respect to the ground level, $n_{o}$, of each ion. If the gas is to be ionized and excited then $\rho u \mathrm{erg} \mathrm{cm}^{-3}$ must be added to the gas. Of this amount $E_{s i}$ strips the atoms and ions of their electrons, $E_{s e}$ promotes the excitation of electrons in the atoms/ions and the reminder brings the system to a common temperature $T$.

The plasma pressure is

$p=\left(n_{\mathrm{tot}}+n_{e}\right) k_{\mathrm{B}} T=n_{\mathrm{tot}}(1+\langle Z\rangle) k_{\mathrm{B}} T=\frac{\rho}{\mu m_{u}}(1+\langle Z\rangle) k_{\mathrm{B}} T$,

where $\langle Z\rangle=n_{e} / n_{\text {tot }}$ and $\mu$ are the mean electron charge and mean molecular weight, respectively. From Eqs. (5) and (9), it is seen that

$p=\frac{2}{3} \rho u-E_{s i}-E_{s e}$,

which is different than the classical relation $p=(\gamma-1) \rho u$ (see, e.g., Vandenbroucke et al. 2013). Consequently, the adiabatic parameter $\gamma=(\partial \ln p / \partial \ln \rho)_{s}$ depends on the state of the plasma through the internal energy. It can be written as (Cox \& Giuli 1968)

$\gamma=\frac{1}{c_{V}}\left(\frac{p}{T \rho}\right) \chi_{T}^{2}+\chi_{\rho}$

with $c_{\mathrm{V}}=(\partial u / \partial T)_{\mathrm{V}}\left(\operatorname{erg~g}^{-1} \mathrm{~K}^{-1}\right)$ denoting the specific heat at constant volume, and the coefficients $\chi_{T}$ and $\chi_{\rho}$ (temperature and density exponents) are written in terms of $\mu$ as

$\chi_{T}=\frac{T}{p}\left(\frac{\partial p}{\partial T}\right)_{\rho}=1-\frac{T}{\mu}\left(\frac{\partial \mu}{\partial T}\right)_{\rho}$

and

$\chi_{\rho}=\frac{\rho}{p}\left(\frac{\partial p}{\partial \rho}\right)_{T}=1-\frac{\rho}{\mu}\left(\frac{\partial \mu}{\partial \rho}\right)_{T}$.

\section{Thermal model}

In order to study the evolution of $\gamma$ with temperature, we follow the evolution of a gas parcel freely cooling from $10^{9} \mathrm{~K}$ (where it is assumed to be completely ionized) evolving in CIE. The gas parcel is composed of the ten most abundant elements in Nature $(\mathrm{H}, \mathrm{He}, \mathrm{C}, \mathrm{N}, \mathrm{O}, \mathrm{Ne}, \mathrm{Mg}, \mathrm{Si}, \mathrm{S}$, and $\mathrm{Fe}$ ) having solar abundances. These are based on Asplund et al. (2009) and include the updates to the $\mathrm{Mg}, \mathrm{Si}, \mathrm{S}$, and Fe abundances by Scott et al. (2015b,a; see also Amarsi \& Asplund 2017 for the abundance of Si). The physical processes included in these calculations comprise electron impact ionization (including excitation-autoionization), radiative and dielectronic recombinations and line excitation by electrons assuming the coronal approximation. In these calculations line excitation/deexcitation by protons, continuum emission, and charge exchange reactions are not included.

\subsection{Ionic fractions}

The time-independent evolution of the ion fractions due to the 102 ions and 10 atoms (in a total of 112 equations) where ionization and recombinations of ions of nuclear charge $Z$ occur between neighbouring ionization stages $z-1, z$, and $z+1$, is given by

$\mathcal{I}_{Z, z-1} x_{Z, z-1} n_{e}-\left(\mathcal{I}_{Z, z}+\mathcal{R}_{Z, z}\right) x_{Z, z} n_{e}+\mathcal{R}_{Z, z+1} x_{Z, z+1} n_{e}=0$,

where $\mathcal{R}_{Z, z}$ and $\mathcal{I}_{Z, z}$ are the rates of recombination and ionization from state $(Z, z)$ to $(Z, z-1)$ and $(Z, z+1)$, respectively; $n_{e}$ is the electron density and $x_{Z, z}=n_{Z, z} / n_{Z}$ the ionic fraction of element $Z$ with effective charge $z$. The ion density is then given by

$n_{Z, z}=x_{Z, z} n_{Z}=x_{Z, z} A(Z) n_{H}$,

with $A(Z)=n_{Z} / n_{H}$ and $n_{H}$ being the abundance of element $Z$ and the hydrogen density. The system of equations can be cast into the matrix equation

$A X=0$,

where $X$ is a vector comprising all ion fractions $x_{Z, z}$ and $A$ is a tridiagonal matrix with elements $\mathcal{I}_{Z, z-1} n_{e},-\left(\mathcal{I}_{Z, z}+\mathcal{R}_{Z, z}\right) n_{e}$, and $\mathcal{R}_{Z, z+1} n_{e}$ at each row populating the diagonal band.

\subsection{Ionization and recombination rates}

From the electron impact ionization cross sections, we calculate the corresponding ionization rates associated with an ion of atomic number $Z$ and ionic charge $z$ by averaging the product $\sigma(E) v$ over the impacting particle kinetic energy distribution $f(E)$ :

$\langle\sigma v\rangle=\int_{I_{z, z}}^{+\infty} \sigma(E)\left(2 E / m_{e}\right)^{1 / 2} f(E) \mathrm{d} E \mathrm{~cm}^{3} \mathrm{~s}^{-1}$,

where $m_{e}$ is the electron mass, and $I_{z, z}$ is the threshold energy in $\mathrm{eV}$.

The radiative and dielectronic recombination rates for the $n$ distribution are calculated from the fit coefficients to the Maxwellian rates (Dzif̌́áková 1998), that is, the radiative recombination rates for the $n$-distribution are determined from

$\alpha_{R R}^{n}=\alpha_{Z, z}^{M B} B_{n} \frac{\Gamma(n / 2-\eta+1)}{\Gamma(3 / 2-\eta)}$, 
where $\alpha_{R R}^{M B}$ is the Maxwellian radiative recombination rate, and $\eta$ is a parameter of the power-law fit of the Maxwellian rate (Woods et al. 1981):

$\alpha_{R R}^{M B}=A_{\text {rad }}\left(\frac{T}{10^{4} \mathrm{~K}}\right)^{-\eta} \mathrm{cm}^{3} \mathrm{~s}^{-1}$.

The dielectronic recombination rates for the $n$-distribution of electrons are determined from coefficients of the Maxwellian rates given by the Burgess (1965) general formula

$\alpha_{D R}^{M B}=\frac{1}{\left(k_{\mathrm{B}} T\right)^{3 / 2}} \sum_{j} c_{j} \mathrm{e}^{-E_{j} /\left(k_{\mathrm{B}} T\right)} \mathrm{cm}^{3} \mathrm{~s}^{-1}$

through (Dzifčáková 1998):

$\alpha_{D R}^{n}=\frac{B_{n}}{\left(k_{\mathrm{B}} T\right)^{3 / 2}} \sum_{j} c_{j}\left(\frac{E_{j}}{k_{\mathrm{B}} T}\right)^{(n-1) / 2} e^{-E_{j} /\left(k_{\mathrm{B}} T\right)} \mathrm{cm}^{3} \mathrm{~s}^{-1}$.

In these expressions $A_{\text {rad }}$ and $c_{j}$ are fitting coefficients.

The radiative and dielectronic recombination rates for the $\kappa$ distribution are also based on the Maxwellian rates and are expressed, respectively, by (Wannawichian et al. 2003; Dzifčáková \& Dudík 2013; see also Dzifčáková 1992)

$\alpha_{R R}^{\kappa}=\alpha_{Z, z}^{M B} A_{\kappa} \frac{\Gamma(\kappa+\eta-1 / 2)}{\Gamma(\kappa+1)}(\kappa-3 / 2)^{-\eta+3 / 2} \mathrm{~cm}^{3} \mathrm{~s}^{-1}$

and

$\alpha_{D R}^{\kappa}=\frac{A_{\kappa}}{\left(k_{\mathrm{B}} T\right)^{3 / 2}} \sum_{j} c_{j}\left[1+\frac{E_{j}}{(\kappa-3 / 2) k_{\mathrm{B}} T}\right]^{-(\kappa+1)} \mathrm{cm}^{3} \mathrm{~s}^{-1}$,

\subsection{Levels populations and excitation rates}

The levels populations are calculated assuming excitationdeexcitation equilibrium, that is, equilibrium between the excitations (by particle impact) and deexcitations (by particle impact and spontaneous decays) to and from a level. We assume that the excitations and deexcitations by particle impact are only due to electrons. The population of level $j$ is determined from the collisional excitations of electrons from levels $m$ to level $j$ ( $m<j$; excitation rate $\left.C_{m j}^{e}\right)$ and from level $j$ to levels $n(j<n$; excitation rate $C_{j n}^{e}$ ), and deexcitations from levels $n$ to level $j$ ( $n>j$; collisional deexcitation rates $C_{n j}^{d}$ and spontaneous decay rates $A_{n j}$ ) and from level $j$ to levels $m$ ( $j>m$; collisional deexcitation and spontaneous decay rates $C_{j m}^{d}$ and $A_{j m}$, respectively). The ground level is populated/depopulated by the deexcitations/excitations from/to levels above it. Thus, the population of a level $j$ is given by the solution of (Phillips et al. 2008)

$$
\begin{aligned}
\sum_{m<j} C_{m j}^{e} n_{e} n_{Z, z, m}+\sum_{n>j}\left(A_{n j}+C_{n j}^{d} n_{e}\right) n_{Z, z, n} \\
-n_{Z, z, j}\left[\sum_{j<n} C_{j n}^{e} n_{e}+\sum_{j>m}\left(A_{j m}+C_{j m}^{d} n_{e}\right)\right]=0,
\end{aligned}
$$

coupled to the equation of mass conservation

$\sum_{j} n_{Z, z, j}=n_{Z, z}$
In these equations $n_{Z, z, m}, n_{Z, z, j}, n_{Z, z, n}$ denote the population densities (in units of $\mathrm{cm}^{-3}$ ) of levels $m, j$, and $n$, respectively; $n_{e}$ and $n_{Z, z}$ are the electron and ion densities. The units of the excitation/deexcitation rates are in $\mathrm{cm}^{3} \mathrm{~s}^{-1}$, while those of the spontaneous decay rates are in $\mathrm{s}^{-1}$.

The rates of collisional excitation from level $i$ to level $j$ $(i<j)$ and deexcitation from level $j$ to level $i$ are determined by the average of the corresponding cross sections $\left(\sigma_{i j}\left(E_{e}\right)\right.$ and $\left.\sigma_{j i}\left(E_{e}^{\prime}\right)\right)$ over all possible velocities of the incident electrons, that is, $C_{i j}^{e}=\left\langle\sigma_{i j}\left(E_{e}\right) v\right\rangle$ and $C_{j i}^{d}=\left\langle\sigma_{j i}\left(E_{e}^{\prime}\right) v^{\prime}\right\rangle$, respectively. The excitation cross section can be written in terms of the dimensionless collision strength $\Omega_{i j}$ through the expressions

$\sigma_{i j}\left(E_{e}\right)=\pi a_{0}^{2} \frac{E_{H}}{\omega_{i} E_{e}} \Omega_{i j}\left(E_{e}\right)=\pi a_{0}^{2} \frac{E_{e}}{\omega_{i} E_{i j}} \frac{\Omega_{i j}\left(E_{e}\right)}{U}$,

where $a_{0}$ is the Bohr radius, $E_{H}$ is the ground state energy of the hydrogen atom, $E_{e}$ is the energy of the incident electron, $\omega_{i}$ is the statistical weight of levels $i$ and $j, E_{i j}$ is the excitation energy between levels $i$, and $U=E_{e} / E_{i j}$ is the reduced electron energy. The collision strength is symmetrical with respect to the direct and the inverse processes, that is, $\Omega_{i j}\left(E_{e}\right)=\Omega_{j i}\left(E_{e}^{\prime}\right)$. Therefore, the deexcitation cross section can be written as

$\sigma_{j i}\left(E_{e}^{\prime}\right)=\pi a_{0}^{2} \frac{E_{e}^{\prime}}{\omega_{j} E_{i j}} \frac{\Omega_{i j}\left(E_{e}\right)}{U^{\prime}}$.

In this expression $\omega_{j}$ is the statistical weight of level $j, E_{e}^{\prime}$ is the energy of the electron impacting on the electrons in level $j$ and $U^{\prime}=E_{e}^{\prime} / E_{i j}$ is the reduced electron energy.

For electrons with a $\mathrm{MB}, \kappa$ and $n$ distributions, the rates of collisional excitation between levels $i$ and $j$ and deexcitation between levels $j$ and $i$ are given by (see, for example, Dudík et al. 2014)

$C_{i j}^{e}=8.629 \times 10^{-6} T^{-1 / 2} \omega_{i}^{-1} \mathrm{e}^{-y} \Upsilon_{i j}(T)$

and

$C_{j i}^{e}=8.629 \times 10^{-6} T^{-1 / 2} \omega_{j}^{-1} d_{j i}(T)$,

respectively. In these expressions $y=E_{i j} / k_{\mathrm{B}} T, \omega_{i}$ and $\omega_{j}$ are the statistical weights of levels $i$ and $j$, respectively, and $T$ is the temperature (in degrees $\mathrm{K}$ ). The $\Upsilon_{i j}(T)$ (also known as the effective collision strength or Upsilon) and $d_{j i}(T)$ (also known as Downsilon) have different forms according to the electron distribution. They are given by

$\Upsilon_{i j}(T)=y e^{y} \int_{1}^{+\infty} \Omega_{i j}(U) \mathrm{e}^{-y U} \mathrm{~d} U$,
$\varkappa_{j i}(T)=y \int_{0}^{+\infty} \Omega_{j i}\left(U^{\prime}\right) \mathrm{e}^{-y U^{\prime}} \mathrm{d} U^{\prime}$,

for thermal,

$\Upsilon_{i j}(T)=y \mathrm{e}^{y} \int_{1}^{+\infty} \Omega_{i j}(U) \frac{A_{\kappa}}{\left(1+\frac{y U}{\kappa-3 / 2}\right)^{\kappa+1}} \mathrm{~d} U$,
$J_{j i}(T)=y \int_{0}^{+\infty} \Omega_{j i}\left(U^{\prime}\right) \frac{A_{\kappa}}{\left(1+\frac{y U^{\prime}}{\kappa-3 / 2}\right)^{\kappa+1}} \mathrm{~d} U^{\prime}$,

for $\kappa$, and

$\Upsilon_{i j}(T)=y \mathrm{e}^{y} B_{n} \int_{1}^{\infty} \Omega_{i j}(U)(y U)^{(n-1) / 2} \mathrm{e}^{-y U} \mathrm{~d} U$, 
$d_{j i}(T)=y B_{n} \int_{0}^{+\infty} \Omega_{j i}\left(U^{\prime}\right)\left(y U^{\prime}\right)^{(n-1) / 2} \mathrm{e}^{-y U^{\prime}} \mathrm{d} U^{\prime}$,

for $n$ distributed electrons. It should be noticed from the above expressions that $\Upsilon_{i j}(T) \neq d_{j i}(T)$ for the non-Maxwellian distributions.

$\Omega_{i j}(U)$ can be written with the functional (Mewe 1972; Mewe \& Gronenschild 1981; Clark et al. 1982; Suno \& Kato 2006; Dzifčáková 2006; Dzifčáková et al. 2015)

$\Omega_{i j}(U)=A_{0}+\sum_{n=1}^{n_{\max }} \frac{A_{n}}{U^{n}}+D \ln U$.

Making use of the exponential integral of order $n, E_{n}(y)$ (Abramowitz \& Stegun 1972) ${ }^{1}$, the integral in the RHS of (30) becomes

$$
\begin{aligned}
\int_{1}^{\infty} \Omega_{i j}(U) \mathrm{e}^{-y U} \mathrm{~d} U & =\int_{1}^{\infty}\left[A_{0}+\sum_{n=1}^{n_{\max }} \frac{A_{n}}{U^{n}}+D \ln U\right] \mathrm{e}^{-y U} \mathrm{~d} U \\
& =A_{0} \frac{e^{-y}}{y}+\sum_{n=1}^{n_{\max }}\left[A_{n} E_{n}(y)\right]+\frac{D}{y} E_{1}(y),
\end{aligned}
$$

and $\Upsilon_{i j}$ is given by

$\Upsilon_{i j}=A_{0}+y \sum_{n=1}^{n_{\max }}\left(A_{n} \beta_{n}\right)+D \beta_{1}$,

with $\beta_{n}=e^{y} E_{n}(y)$. We can use this result to fit the available $\Upsilon_{i j}$ and thus obtain the corresponding collision strength through the determination of the best-fit parameters $A_{0}, \ldots, A_{n_{\max }}$ and $D$ (Dzifčáková 2006; Dzif̌cáková et al. 2015). This procedure is used because most of the theoretical collision strengths are not made available by their authors, instead what is available are the effective collision strengths calculated with the MB distribution.

In the present calculations we assumed an atomic/ionic model composed of 30 levels and used the procedure discussed above with $n_{\max }=8$ to determine the collision strengths of the transitions (see further details in de Avillez \& Anela, in prep.).

\subsection{Atomic data}

Electron impact ionization cross sections discussed in Dere (2007) and available in the Chianti database (see, e.g., Dere et al. 2009) are adopted.

The Maxwellian radiative recombination rate coefficients are taken from Badnell $(2006 \mathrm{c})^{2}$ for all bare nuclei through $\mathrm{Na}$ like ions recombining to $\mathrm{H}$ through $\mathrm{Mg}$-like ions, Altun et al. (2007) for Mg-like ions, Abdel-Naby et al. (2012) for Al-like ions, Nikolić et al. (2010) for Ar-like ions, and Badnell (2006b) for FeXIII-FeXions. The Maxwellian dielectronic recombination rates are taken from Badnell (2006a) or H-like ions, Bautista \& Badnell (2007) for He-like ions, Colgan et al. (2003, 2004) for

1 The exponential integral of order $n$ is

$E_{n}(y)=\int_{1}^{\infty} \frac{\mathrm{e}^{-t y}}{t^{n}} \mathrm{~d} t$, with $n>0$.

$E_{n+1}(y)$ can be determined through the recurrence formula

$e^{y} E_{n+1}=\frac{1}{n}\left(1-y e^{y} E_{n}(y)\right)$.

2 http://amdpp.phys.strath.ac.uk/tamoc/DATA/
$\mathrm{Li}$ and Be-like ions, Altun et al. (2004, 2006, 2007) for B, Na, and Mg-like ions, Zatsarinny et al. (2003, 2004a,b, 2006) for C, O, F, and Ne-like ions, Mitnik \& Badnell (2004) for N-like ions, Abdel-Naby et al. (2012) for Al-like ions, and Nikolić et al. (2010) for Ar-like ions. We further used the data in the errata by Altun et al. (2005) for NeVI and MgVII, Zatsarinny et al. (2005a) for NII, and Zatsarinny et al. (2005b) for NeIII, MgV, and FeXIX.

Radiative and dielectronic recombination rates for SII, SIII, and FeVII are adopted from Mazzotta et al. (1998), while for the remaining ions we adopt the radiative and dielectronic recombination rates derived with the unified electron-ion recombination method (Nahar \& Pradhan 1994) and available at NORADAtomic-Data ${ }^{3}$.

The wavelengths, Einstein coefficients of spontaneous transitions and the effective collision strengths of transitions calculated for a MB electron distributions are taken from version 8.0.7 of the CHIANTI atomic database ${ }^{4}$ (Del Zanna et al. 2015).

\subsection{Numerical methods and calculations}

The ionic structure of the plasma is determined by solving the system of Eq. (14) at each temperature using a Gauss elimination method with scaled partial pivoting (Cheney \& Kincaid 2008) and a tolerance of $10^{-15}$. The numerical integrations in Eq. (17) are carried out with a precision of $10^{-15}$ using the double-exponential over a semi-finite interval method of Takahasi \& Mori (1974) and Mori \& Sugihara (2001). We use a modified version (parallelized version) of the Numerical Automatic Integrator for Improper Integral package developed by T. Ooura ${ }^{5}$.

The calculations proceed as follows: follow the evolution of a gas parcel freely evolving isochorically under CIE from the initial conditions (fully ionized plasma at $10^{9} \mathrm{~K}$ with a density $\left.n_{H}=1.0 \mathrm{~cm}^{-3}\right)$. At each temperature the electron density, ionization and recombination rates and the ionic fractions are determined first. This is followed by the determination of the collisional excitation rates and of the populations of the excited states given by Eq. (24). Finally, the mean molecular weight, the pressure, the internal energy, the specific heat at constant volume $c_{v}$ and the gamma parameter are calculated.

\section{Results}

\subsection{Internal energy}

The total specific internal energy ( $\mathrm{erg} \mathrm{g}^{-1}$ ) of a plasma evolving under CIE for the MB, $n$ and $\kappa$ distributions, using $n, \kappa=2,3,5$, 10, and 15 are displayed in Fig. 2, while its thermal and ionization components are shown in Fig. 3. The excitation component of the internal energy has less than a percent contribution to the total energy and thus, is not shown in the figures. The top and bottom panels in both figures refer to plasmas with, respectively, $n$ and $\kappa$ electron distributions. Maxwellian energies are shown by the black lines and dots in the top and bottom panels of both figures.

The total internal energy (as well as the thermal, ionization, and excitation components) of the gas varies according to the parameters $\kappa$ and $n$. As $\kappa$ increases ( $n$ increases), the internal energy approaches (moves away of) that obtained for a Maxwellian distribution of electrons. The largest variations

\footnotetext{
3 http://www . astronomy . ohio-state.edu/ nahar/

4 http://www . chiantidatabase.org

5 http://www.kurims.kyoto-u.ac.jp/ ooura/intde.html
} 

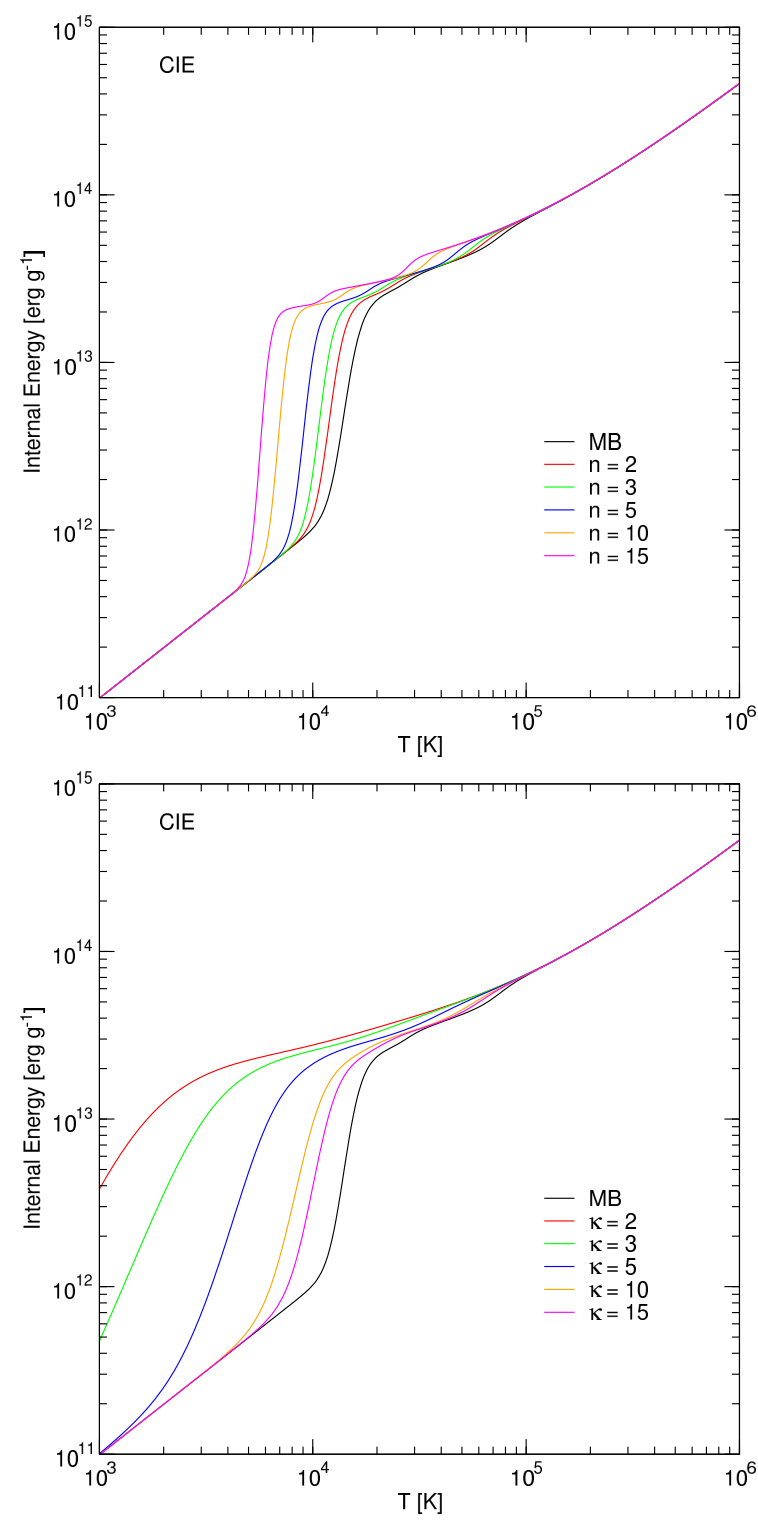

Fig. 2. Total specific internal energy of the plasma for MaxwellBoltzmann (black line in both panels), $n$ (top panel), and $\kappa$ (bottom panel) distributions calculated for $n, \kappa=2, \ldots, 15$.

in the total internal energy occur in $\kappa$ distributed plasmas and are mainly driven by the large variations (more than an order of magnitude with regard the thermal component for $T<10^{4} \mathrm{~K}$ ) in the ionization energy. In $n$ distributed plasmas the energies (total, thermal and ionization) profiles are similar but with a shift to the left as $n$ increases (top panels of both figures). In the $n$-distributed plasma the dominance of the ionization energy over the thermal component only occurs down to the temperature at which the ionization energy has a sharp decrease. Below that temperature the thermal energy dominates (top panel of Fig. 3).

\subsection{The adiabatic parameter}

Figure 4 displays the $\gamma$ parameter calculated for a monoatomic Maxwellian plasma composed of (1) $\mathrm{H}$ (black line), (2) $\mathrm{H}$ and $\mathrm{He}$ (red line), (3) metals (C, N, O, Ne, Mg, Si, S, and Fe; green line), and (4) all elements ( $\mathrm{H}, \mathrm{He}$, and metals; black dashed line). The adiabatic parameter is $5 / 3$ at $T \leq 8000 \mathrm{~K}$ (cases 1,2 , and 4),
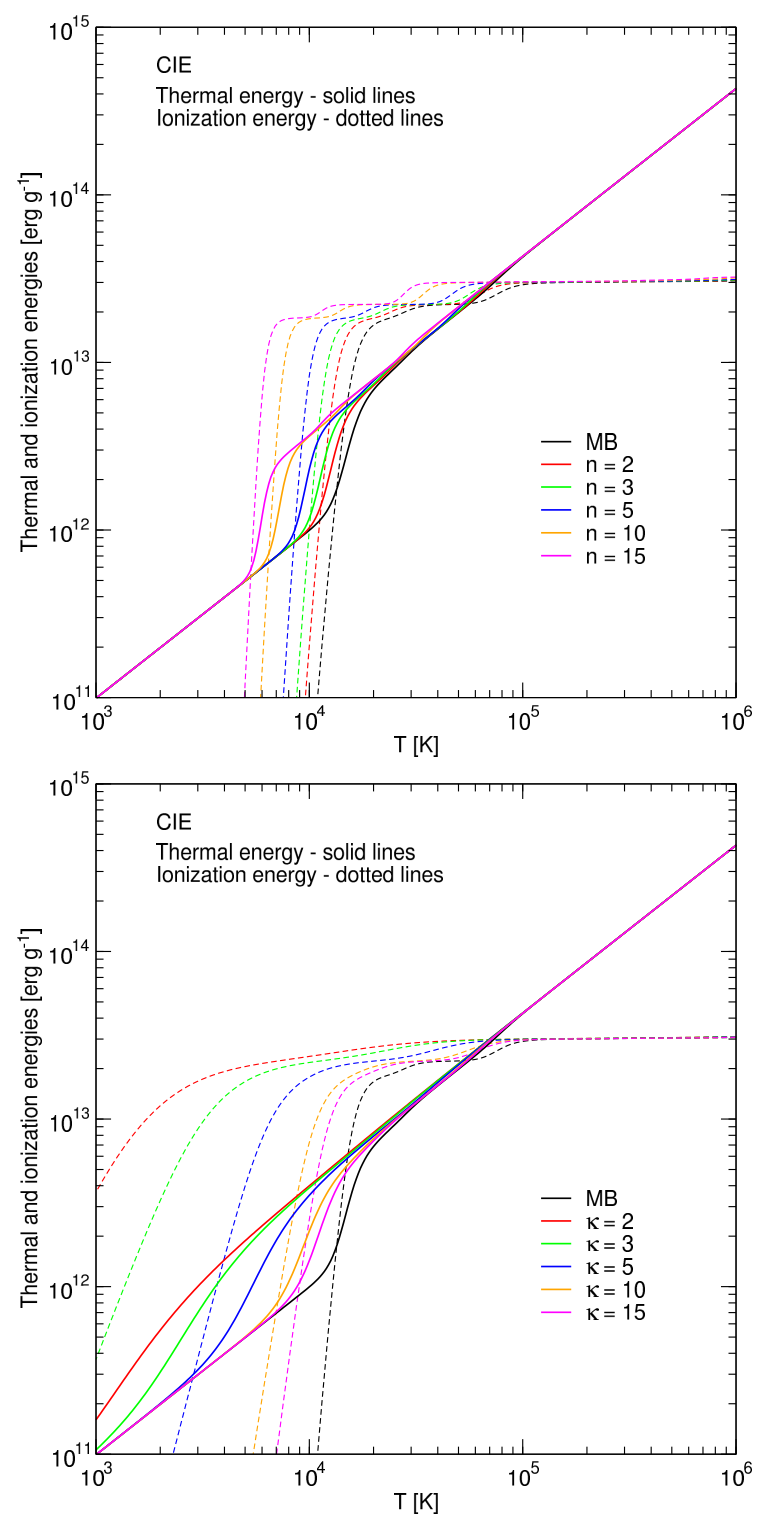

Fig. 3. Specific thermal and ionization energies of the plasma for Maxwell-Boltzmann (black solid and dashed lines in both panels), $n$ (top panel), and $\kappa$ (bottom panel) distributions with $n, \kappa=2, \ldots, 15$.

at $T \leq 3090 \mathrm{~K}$ (case 3 ) and at $T \geq 5 \times 10^{4} \mathrm{~K}$ (case 1$), 2 \times 10^{5} \mathrm{~K}$ (case 2 ), $5 \times 10^{6} \mathrm{~K}$ (case 4 ), and $4 \times 10^{8} \mathrm{~K}$ (case 3 ). In the remaining temperatures, $\gamma$ has substantial variations having values as low as 1.08 (cases 1, 2, and 4) and 1.1 (case 3 ). In the mixtures comprising $\mathrm{H}, \mathrm{He}$, and metals the former two determine the value of $\gamma$ as they have abundances much larger than the remaining elements. Thus, the three transitions observed in case (4) are determined from left to right (from low to high temperatures) by the HI, HeI, and HeII ionizations, respectively. In case (3) the observed transitions in the $\gamma$ profile are due to the ionization of the most abundant elements among the metals.

Figure 5 displays the temperature variation of the adiabatic parameter in plasmas with $n$ (top and middle panels) and $\kappa$ (bottom panel) electron distributions and composed of a mixture of $\mathrm{H}, \mathrm{He}$, and metals (case 4) for $n, \kappa=2, \ldots, 15$. The Maxwellian evolution of $\gamma$ is shown by the black dashed lines. As $n$ increases, $\gamma$ moves towards the left of the Maxwellian value with the same number of transitions (a result of the dominance of the ionization by $\mathrm{H}$ and $\mathrm{He}$ due to their abundances) but shifted to lower 


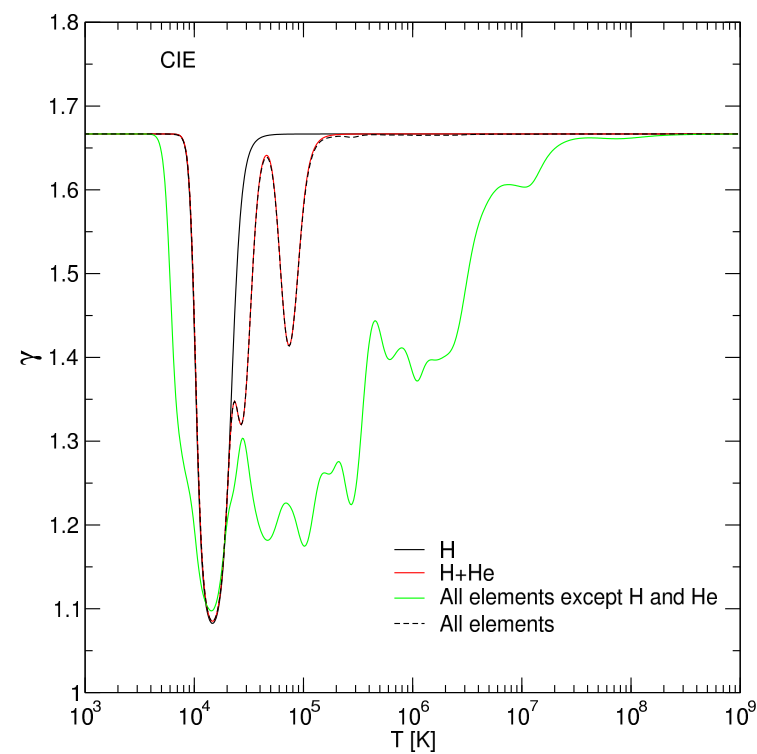

Fig. 4. Evolution of the $\gamma$ parameter in different plasmas composed only of $\mathrm{H}$ (black line), $\mathrm{H}$ and $\mathrm{He}$ (red line), metals (C, N, O, Ne, Mg, Si, S, and $\mathrm{Fe}$; green line), and all elements (black dashed line). Note that $\mathrm{H}$ and He determine the evolution of $\gamma$ for the calculations involving all the ten elements because the abundances of the metals are much smaller than those of $\mathrm{H}$ and $\mathrm{He}$.

temperatures than those observed for lower $n$. The local minimum seen after each transition becomes deeper with increasing $n$. Therefore, the minimum value of $\gamma$ decreases from 1.09 for the Maxwellian distributed plasma to 1.025 for $n=15$ passing by 1.05 for $n=5$. That is, with increasing $n$, the plasma behaves almost isothermally at that range for temperatures.

In a $\kappa$ distributed plasma, as $\kappa$ increases $\gamma$ approaches the Maxwellian value (bottom panel of Fig. 5). While the Maxwellian $\gamma$ has three transitions, the $\kappa$ distributed $\gamma$ only has three transitions for $\kappa>15$. Over the remaining values of $\kappa$ two transitions are seen for $\kappa>4$ and no transitions are seen at lower $\kappa$. The latter is a consequence of the early ionization of $\mathrm{H}$ and He when $\kappa$ has small values as can be seen in Fig. 6, which displays the ionization rates of $\mathrm{H}, \mathrm{He}$, and $\mathrm{He}^{+}$, thereby affecting the internal energy of the plasma. As $\kappa$ increases, the minimum value of $\gamma$ increases from almost $1.01(\kappa=2)$ to 1.09 as $\kappa \rightarrow \infty$, that is, the Maxwellian value. $\gamma=5 / 3$ at $T>2 \times 10^{5} \mathrm{~K}$ for all possible values of $\kappa$, while at $T<8000 \mathrm{~K} \gamma<5 / 3$ for $\kappa<7$.

\section{Final remarks}

The calculations presented in this paper comprise the determination of the $\gamma$ parameter in a plasma characterized by $\mathrm{MB}, n$ and $\kappa$ electron distributions and evolving under collisional ionization equilibrium and cooling from $10^{9} \mathrm{~K}$. Two particle processes (electron impact ionization including excitation-autoionization, radiative and dielectronic recombination and excitation) are considered in all the calculations. The populations of excited states are calculated assuming the coronal approximation. It is shown that the $\gamma$ parameter in an optically thin monoatomic plasma depends on its ionic state and most importantly on the contribution of the ionization energy to the total internal energy. The excitation energy has a negligible contribution to the total internal energy. Lookup tables of the temperature variation of $\gamma$ are provided as supplementary material and can be used in simulations of solar, interstellar or intergalactic plasmas.
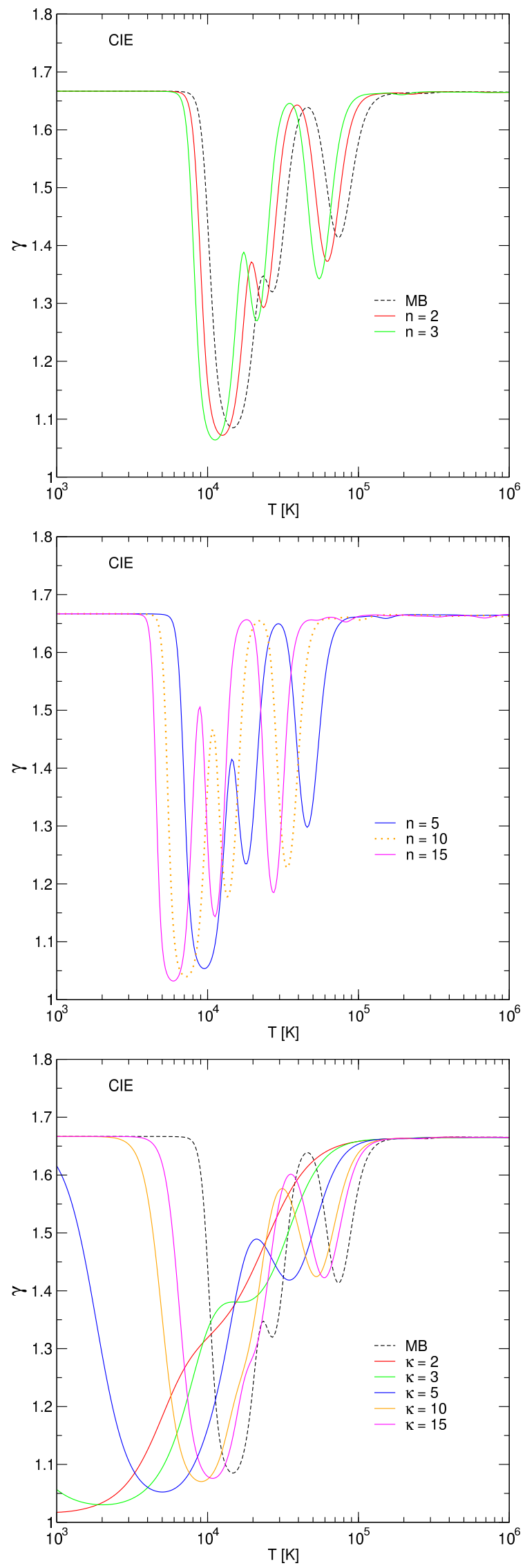

Fig. 5. Evolution with temperature of the adiabatic parameter $(\gamma)$ of a plasma evolving under CIE for Maxwell-Boltzmann (black line in the top and bottom panels), $n$ (top and middle panels), and $\kappa$ (bottom panel), distributions calculated for $n, \kappa=2,3,5,10$, and 15 . 

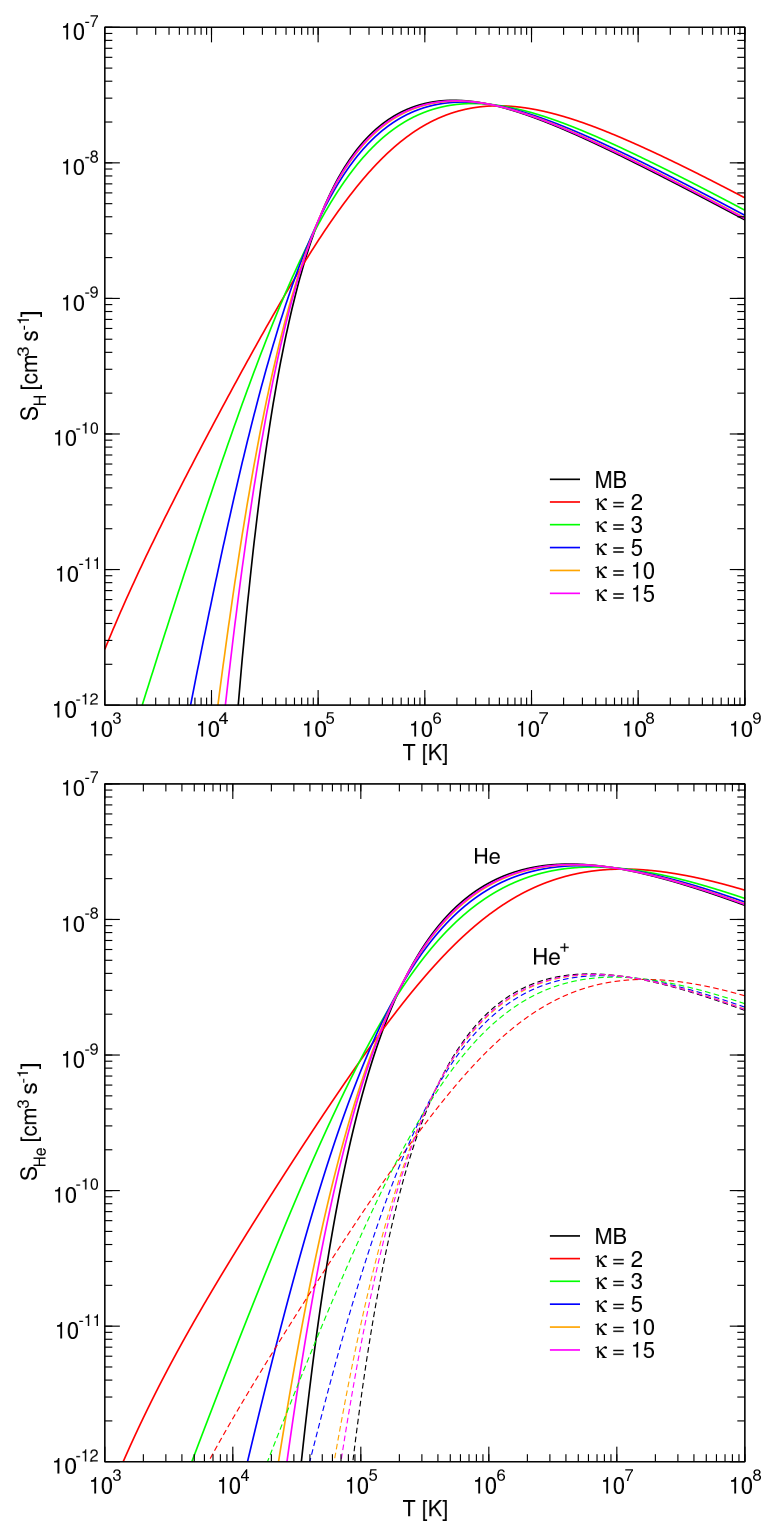

Fig. 6. Variation with $\kappa$ of the ionization rates of $\mathrm{H}, \mathrm{He}$, and $\mathrm{He}^{+}$.

Although CIE cooling functions are widely used in interstellar medium simulations, caution should be exercised as CIE is only valid provided the cooling timescale $\left(\tau_{\text {cool }}\right)$ of the plasma is larger than the recombination time scales of the different ions $\left(\tau_{\text {rec }}^{Z, z}\right)$, something that typically occurs at $T>10^{6} \mathrm{~K}$. For lower temperatures, $\tau_{\text {cool }}<\tau_{\text {rec }}^{Z, z}$, and therefore, cooling and recombination are not synchronized, and the plasma appears overionized, if it is cooling (for detailed discussions, see, e.g., Kafatos 1973; Shapiro \& Moore 1976; Sutherland \& Dopita 1993; Schmutzler \& Tscharnuter 1993; Gnat \& Sternberg 2007; de Avillez \& Breitschwerdt 2010, and references therein). Consequently, the history of each gas parcel, evolving from different initial conditions, will have different ionic/radiative histories. Something that does not happen with CIE.

Futhermore, de Avillez \& Breitschwerdt (2012) have shown in a muti-fluid time-dependent study of the dynamical and thermal evolutions of the interstellar gas ${ }^{6}$ that this historyreflects

\footnotetext{
6 Hydrodynamical multi-fluid simulations of the interstellar medium in the disk and halo of the Milky Way, including the time-dependent evolution of all atoms and ions associated with the ten most abundant elements in Nature (H, He, C, N, O, Ne, Mg, Si, S, and Fe).
}

the feedback between heating, cooling and the dynamics of the system. Therefore, even with the same initial conditions the gas parcels will have a different ionic/radiative history with the corresponding emissivities having an order of magnitude differences.

We have shown that widely performed hydro- and magnetohydrodynamical simulations of optically thin plasmas like in the interstellar and intergalactic medium should take into account not only the time-dependent ionization structure (see de Avillez \& Breitschwerdt 2012) but also the detailed distribution between internal and potential energies leading to different values of the ratio of specific heats, $\gamma$. In addition, it should be checked during simulations if MB equilibrium is established or if more generalized forms of electron distributions like $\kappa$ or $n$ are more applicable.

In a forthcoming paper, we describe the evolution of the $\gamma$ parameter under non-equilibrium ionization conditions and with molecular contributions to the ionization state of the plasma as they affect the $\gamma$ parameter at low temperatures (see, e.g., Decampli et al. 1978; Wuchterl 1991; D’Angelo \& Bodenheimer 2013).

Acknowledgements. We thank the anonymous referee for the comments improving the paper. This research was supported by the project Enabling Green E-science for the SKA Research Infrastructure (ENGAGE SKA), reference POCI-01-0145-FEDER-022217, funded by COMPETE 2020 and FCT, Portugal. D.B. acknowledges support from the DeutscheForschungsgemeinschaft, DFG project ISM-SPP 1573. The calculations were carried out at the ISM - Xeon Phi cluster of the Computational Astrophysics Group, University of Évora, acquired under project "Hybrid computing using accelerators \& coprocessorsmodelling nature with a novell approach" (PI: M.A.), InAlentejo program, CCDRA, Portugal.

\section{References}

Abdel-Naby, S. A., Nikolić, D., Gorczyca, T. W., Korista, K. T., \& Badnell, N. R. 2012, A\&A, 537, A40

Abramowitz, M., \& Stegun, I. A. 1972, Handbook of Mathematical Functions (New York: Dover Publications, Inc.)

Altun, Z., Yumak, A., Badnell, N. R., Colgan, J., \& Pindzola, M. S. 2004, A\&A, 420, 775

Altun, Z., Yumak, A., Badnell, N. R., Colgan, J., \& Pindzola, M. S. 2005, A\&A 433, 395

Altun, Z., Yumak, A., Badnell, N. R., Loch, S. D., \& Pindzola, M. S. 2006, A\&A, 447, 1165

Altun, Z., Yumak, A., Yavuz, I., et al. 2007, A\&A, 474, 1051

Amarsi, A. M., \& Asplund, M. 2017, MNRAS, 464, 264

Asplund, M., Grevesse, N., Sauval, A. J., \& Scott, P. 2009, ARA\&A, 47, 481

Badnell, N. R. 2006a, A\&A, 447, 389

Badnell, N. R. 2006b, ApJ, 651, L73

Badnell, N. R. 2006c, ApJS, 167, 334

Bautista, M. A., \& Badnell, N. R. 2007, A\&A, 466, 755

Behringer, K., \& Fantz, U. 1994, J. Phys. D, 27, 2128

Berezhko, E. G. \& Ellison, D. C. 1999, ApJ, 526, 385

Burgess, A. 1965, ApJ, 141, 1588

Cheney, W., \& Kincaid, D. 2008, Numerical Mathematics and Computing, 6th edn. (Belmont: Thomson Brooks/Cole)

Clark, R. E. H., Magee, Jr., N. H., Mann, J. B., \& Merts, A. L. 1982, ApJ, 254, 412

Colgan, J., Pindzola, M. S., Whiteford, A. D., \& Badnell, N. R. 2003, A\&A, 412, 597

Colgan, J., Pindzola, M. S., \& Badnell, N. R. 2004, A\&A, 417, 1183

Cox, J. P., \& Giuli, R. T. 1968, Principles of Stellar Structure, vol. 1 (Gordon and Breach Science Pub.)

D’Angelo, G., \& Bodenheimer, P. 2013, ApJ, 778, 77

de Avillez, M. A. \& Breitschwerdt, D. 2010, ASP Conf. Ser., 438, 313

de Avillez, M. A., \& Breitschwerdt, D. 2012, ApJ, 756, L3

de Avillez, M. A., \& Breitschwerdt, D. 2015, A\&A, 580, A124

de Avillez, M. A., \& Breitschwerdt, D. 2017, ApJS, 232, 12

Decampli, W. M., Cameron, A. G. W., Bodenheimer, P., \& Black, D. C. 1978, ApJ, 223, 854

Del Zanna, G., Dere, K. P., Young, P. R., Landi, E., \& Mason, H. E. 2015, A\&A, 582, A56 
Dere, K. P. 2007, A\&A, 466, 771

Dere, K. P., Landi, E., Young, P. R., et al. 2009, A\&A, 498, 915

Druyvesteyn, M. J. 1930, Z. Phys., 64, 781

Dudík, J., Del Zanna, G., Mason, H. E., \& Dzifčáková E. 2014, A\&A, 570, A 124

Dzifčáková, E. 1992, Sol. Phys., 140, 247

Dzifčáková, E. 1998, Sol. Phys., 178, 317

Dzifčáková, E. 2006, Sol. Phys., 234, 243

Dzifčáková, E., \& Dudík, J. 2013, ApJS, 206, 6

Dzifčáková, E., Homola, M., \& Dudík, J. 2011, A\&A, 531, A111

Dzifčáková, E., Dudík, J., Kotrč, P., Fárník, F., \& Zemanová, A. 2015 , ApJS, 217,14

Gnat, O., \& Sternberg, A. 2007, ApJS, 168, 213

Hares, J. D., Kilkenny, J. D., Key, M. H., \& Lunney, J. G. 1979, Phys. Rev. Lett., 42, 1216

Humphrey, A., \& Binette, L. 2014, MNRAS, 442, 753

Kafatos, M. 1973, ApJ, 182, 433

Karlický, M., Dzif̌ćáková, E., \& Dudík, J. 2012, A\&A, 537, A36

Livadiotis, G., \& McComas, D. J. 2009, J. Geophys. Res., 114, A11105

Macfarlane, J. J. 1989, Comput. Phys. Commun., 56, 259

Mazzotta, P., Mazzitelli, G., Colafrancesco, S., \& Vittorio, N. 1998, A\&AS, 133, 403

Mewe, R. 1972, A\&A, 20, 215

Mewe, R., \& Gronenschild, E. H. B. M. 1981, A\&AS, 45, 11

Mitnik, D. M. \& Badnell, N. R. 2004, A\&A, 425, 1153

Mori, M. \& Sugihara, M. 2001, J. Comput. Appl. Math., 127, 287

Nahar, S. N. \& Pradhan, A. K. 1994, Phys. Rev. A, 49, 1816

Nicholls, D. C., Dopita, M. A., Sutherland, R. S., Kewley, L. J., \& Palay, E. 2013, ApJS, 207, 21

Nikolić, D., Gorczyca, T. W., Korista, K. T., \& Badnell, N. R. 2010, A\&A, 516, A97

Phillips, K. J. H., Feldman, U., \& Landi, E. 2008, Ultraviolet and X-ray Spectroscopy of the Solar Atmosphere (Cambridge: Cambridge University Press)

Pierrard, V., \& Lazar, M. 2010, Sol. Phys., 267, 153

Porquet, D., Arnaud, M., \& Decourchelle, A. 2001, A\&A, 373, 1110

Schmutzler, T., \& Tscharnuter, W. M. 1993, A\&A, 273, 318
Scott, P., Asplund, M., Grevesse, N., Bergemann, M., \& Sauval, A. J. 2015a, A\&A, 573, A26

Scott, P., Grevesse, N., Asplund, M., et al. 2015b, A\&A, 573, A25

Seely, J. F., Feldman, U., \& Doschek, G. A. 1987, ApJ, 319, 541

Shapiro, P. R. \& Moore, R. T. 1976, ApJ, 207, 460

Suno, H., \& Kato, T. 2006, Atomic Data and Nuclear Data Tables, 92, 407

Sutherland, R. S. \& Dopita, M. A. 1993, ApJS, 88, 253

Takahasi, H., \& Mori, M. 1974, Pub. Res. Inst. Math. Sci., 9, 721

Vaidya, B., Mignone, A., Bodo, G., \& Massaglia, S. 2015, A\&A, 580, A110

Vandenbroucke, B., De Rijcke, S., Schroyen, J., \& Jachowicz, N. 2013, ApJ, 771, 36

Vasyliunas, V. M. 1968, Astrophys. Space Sci. Lib., 10, 622

Wannawichian, S., Ruffolo, D., \& Kartavykh, Y. Y. 2003, ApJS, 146, 443

Woods, D. T., Shull, J. M., \& Sarazin, C. L. 1981, ApJ, 249, 399

Wuchterl, G. 1990, A\&A, 238, 83

Wuchterl, G. 1991, Icarus, 91, 39

Zatsarinny, O., Gorczyca, T. W., Korista, K. T., Badnell, N. R., \& Savin, D. W. 2003, A\&A, 412, 587

Zatsarinny, O., Gorczyca, T. W., Korista, K. T., Badnell, N. R., \& Savin, D. W. 2004a, A\&A, 417, 1173

Zatsarinny, O., Gorczyca, T. W., Korista, K. T., Badnell, N. R., \& Savin, D. W. 2004b, A\&A, 426, 699

Zatsarinny, O., Gorczyca, T. W., Korista, K. T., et al. 2005a, A\&A, 440, 1203

Zatsarinny, O., Gorczyca, T. W., Korista, K. T., et al. 2005b, A\&A, 438, 743

Zatsarinny, O., Gorczyca, T. W., Fu, J., et al. 2006, A\&A, 447, 379

\section{Appendix A: Tables at the CDS}

In the supplementary material, Tables (1 and 2$)$ referring to the adiabatic parameter of the gas versus temperature $(\mathrm{K})$ for the Maxwellian, $n$ and $\kappa$ distributions, with $n, \kappa=2,3,5,10,15$ and 25 , are provided. We note that $n=1$ corresponds to the Maxwellian distribution. More data can be provided by the corresponding author upon request. 